\title{
Prototype Experience for MIMO BLAST Over Third-Generation Wireless System
}

\author{
Ali Adjoudani, Eric C. Beck, Andreas P. Burg, Student Member, IEEE, Goran M. Djuknic, Thomas G. Gvoth, \\ D. Haessig, Senior Member, IEEE, Salim Manji, Student Member, IEEE, Michelle A. Milbrodt, Markus Rupp, \\ Dragan Samardzija, Member, IEEE, Arnold B. Siegel, Member, IEEE, Tod Sizer, II, Member, IEEE, C. Tran, \\ Susan Walker, Stephen A. Wilkus, Member, IEEE, and Peter W. Wolniansky
}

\begin{abstract}
In this paper, a multiple-input-multiple-output (MIMO) extension for a third-generation (3G) wireless system is described. The integration of MIMO concepts within the existing UMTS standard and the associated space-time RAKE receiver are explained. An analysis is followed by a description of an actual experimental MIMO transmitter and receiver architecture, both realized on digital signal processors (DSPs) and FPGAs within a precommercial OneBTS base station. It uses four transmit and four receive antennas to achieve downlink data rates up to $1 \mathrm{Mb} / \mathrm{s}$ per user with a spreading factor of 32 and the UMTS chip rate of 3.84 MHz. Furthermore, different MIMO detectors are evaluated, comparing their performance and complexity. System performance is evaluated through simulations and indoor over-the-air measurements. Capacity and bit-error rate measurement results are presented.
\end{abstract}

Index Terms-OneBTS, rapid prototyping, third-generation (3G), UMTS, wireless multiple-input-multiple-output (MIMO) systems.

\section{INTRODUCTION}

OBILE multimedia and high-speed internet access are driving the development of third-generation $(3 \mathrm{G})$ wireless communication systems toward significantly higher data rates than those achieved by their second-generation $(2 \mathrm{G})$ predecessors. To provide this at a reasonable price, such systems must also support a large number of concurrent users. Using direct sequence code-division multiple access (DS-CDMA), the UMTS standard allows different data rates to be allocated across the active users by appropriate selection of spreading factors, with a maximum data rate of $2 \mathrm{Mb} / \mathrm{s}$. However, assuming quaternary phase-shift keying (QPSK) modulation, the total uncoded data rate of the system is limited to $8 \mathrm{Mb} / \mathrm{s}$. A detailed analysis [1] has

Manuscript received May 1, 2002; revised November 1, 2002.

A. Adjoudani, E. C. Beck, S. Manji, M. A. Milbrodt, D. Samardzija, A. B. Siegel, T. Sizer, II, C. Tran, S. Walker, S. A. Wilkus, and P. W. Wolniansky are with Wireless Research Laboratory, Bell-Labs, Lucent Technologies, Holmdel, NJ 07733 USA (e-mail: ali2@lucent.com; ericbeck@lucent.com; smanji@lucent.com; milbrodt@lucent.com; dragan@lucent.com; siegel@lucent.com; ts2@lucent.com; ct@lucent.com; sue@lucent.com; wilkus@lucent.com; pww@lucent.com).

A. P. Burg is with the Integrated Systems Laboratory, Swiss Federal Institute of Technology (ETH), Zürich, 8092 Switzerland (e-mail: apburg @iis.ee.ethz.ch).

G. M. Djuknic, T. G. Gvoth, and D. Haessig are with Wireless Advance Technologies, Lucent Technologies, Whippany, NJ 07981 USA (e-mail: goran@lucent.com; tgvoth@lucent.com; haessig@ lucent.com).

M. Rupp is with the Institute of Communications and Radio-Frequency Engineering, TU Wien, Vienna, A-1040 Austria (e-mail: mrupp@nt.tuwien.ac.at).

Digital Object Identifier 10.1109/JSAC.2003.809724 shown that these limits are almost never consistently achieved in DS-CDMA systems using conventional RAKE receivers due to multiple-access interference (MAI) and self interference in multipath environments.

The application of multiple antennas is expected to increase system capacity through different mechanisms. The most straight forward approach is to exploit spatial diversity with multiple antennas at the transmitter and/or receiver. Different diversity techniques are used to mitigate the effects of fading and spatially nonwhite interference (in-cell and out-of-cell MAI) thereby increasing the received SINR. As such, one possible method of spatial diversity is already defined within the UMTS standard [2]. In 1996, Foschini pointed out [3] that the use of multiple antennas at the transmitter and at the receiver would allow for a significant increase in system capacity through the application of spatial multiplexing. It is shown that the capacity of such a system with $M$ transmit and $N$ receive antennas is

$$
C=\log _{2}\left[\operatorname{det}\left(\mathbf{I}_{M \times M}+\frac{\mathrm{SNR}_{M}}{M} \mathbf{H H}^{H}\right)\right]
$$

where $\mathbf{H}$ describes the $M \times N$ channel between the $M$ transmit and $N$ receive antennas (the matrix elements are assumed to be complex and unit variance variables). Under idealized conditions (e.g., orthogonal channels and $M=N$ ) this spatial multiplexing results in an $M$ fold capacity gain [4]. A rich scattering environment is known to favor spatial multiplexing. We refer to this form of transmission over multiple antenna systems as Bell Labs layered space-time (BLAST). So far, the concept has been analyzed, demonstrated, and verified for narrowband systems in numerous publications [5]-[10]. A study that compares capacity and bit-error rate (BER) performance of space-time coding, beam forming and BLAST in a UMTS time-division duplex (TDD) scenario has been presented in [11] and [12]. In summary, the authors have concluded that in typical indoor environments and when no reliable channel state information is available at the transmitter [as in the UMTS-frequency-division duplex (FDD) downlink] spatial multiplexing is the method best suited for optimally exploiting the channel capacity.

In this paper, Foschini's concept is applied as an extension to the UMTS FDD downlink. The goal is to provide significantly increased capacity and, thus, higher downlink data rates for a larger number of concurrent users. Only minor modifications to the existing UMTS standard are necessary, allowing this 
form of the multiple-access scheme to co-exist with all regular UMTS users. Moreover, no channel knowledge is required at the transmitter. The rest of this paper is organized as follows. The remainder of the introduction will describe our method for integrating BLAST within the UMTS FDD downlink and the corresponding transmitter and receiver architectures. Further, some theoretical and practical performance aspects will be highlighted. Section II provides a description of the implementation of the main components in the system. Furthermore, the testing of the system and corresponding results are presented and the prototype implementation is described. Section III presents indoor over-the-air BER and capacity measurement results. Before the paper is concluded, as an application example, the implementation of a real-time video streaming system is described.

\section{A. Multiple-input-multiple-output (MIMO) Extended UMTS FDD Downlink}

The design is based on the DS-CDMA principle and uses the UMTS FDD chip-rate of $3.84 \mathrm{MHz}$ [2], [13]. Its essential components are the dedicated physical channels (DPCH), a common pilot channel (CPICH) for the pilot assisted channel estimation, and the primary and secondary synchronization channels (PSCH, SSCH). The common pilot channel, as well as the user channels, are separated by utilizing orthogonal variable rate channelization codes, which are derived from a Hadamard matrix (OVSF-codes). The CPICH uses a length 256 OVSF code, while the length of the codes for the DPCH is variable to support raw data rates between $31.25 \mathrm{~kb} / \mathrm{s}$ and $2 \mathrm{Mb} / \mathrm{s}$ in the single antenna case and $125 \mathrm{~kb} / \mathrm{s}$ to $8 \mathrm{Mb} / \mathrm{s}$ in the proposed $4 \times 4$ antenna configuration. After channelization, the channels are scrambled with the same truncated Gold sequence. Adjacent base stations reside in the same band and are separated through the use of different Gold sequences. The PSCH and the SSCH are added after the scrambling and are not orthogonal to the other codes. They are both required to achieve initial synchronization and to identify the Gold sequence used by the targeted base station [13].

The extension of this basic system to support $M$ transmit and $N$ receive antennas for spatial multiplexing is straight forward. After channel coding, the incoming serial data stream of each user in this mode is first demultiplexed into $M$ parallel streams. Each of the streams uses the same channelization code and is scrambled with the same Gold sequence before it is transmitted through one of the $M$ transmit antennas. The total transmitted power is being conserved by sharing it equally among the antennas and no additional user codes are required. It should also be noted that other users, which do not use this scheme, are not affected, as the codes of the MIMO users are still orthogonal to the other channelization codes in the system. However, while the legacy system requires only one pilot channel for the estimation of the $1 \times 1$ channel at the mobile, this is not sufficient for the pilot assisted estimation of the $M \times N$ channels of the MIMO system. Therefore, a separate pilot channel, again using a length 256 OVSF code is assigned to each of the transmit antennas.

The synchronization channels utilize only one of the transmit antennas, as their replication would lead to an inherent beam forming, which is not intended. Fig. 1 displays the transmitter of the realized system with $M=4$ transmit antennas. Only two

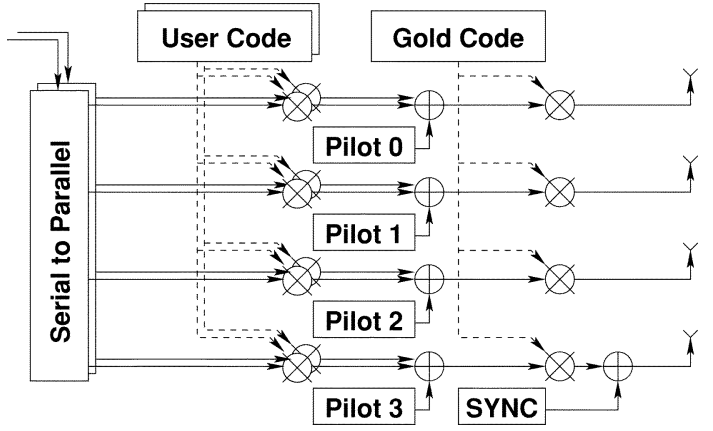

Fig. 1. Transmitter for a MIMO-extended UMTS FDD downlink with four transmit antennas.

users are depicted in this diagram for readability. Many details of the implemented system are presented in [14]-[18].

\section{B. Signal Model and MIMO RAKE Receiver}

It has been shown that large diversity gains are desirable to reduce the effect of fading. It is, therefore, concluded that multipath propagation should be exploited whenever it exists [19]. In general, adding multipath components does not automatically lead to better performance (see the work in [11] and [20]-[21]). In addition, for practical receiver designs multipath is not necessarily a desirable condition as it can also lead to serious multiple access and self interference, reducing the effective system capacity [22]. The severity of the interference depends strongly on the type of receiver. While a MIMO equalizer (temporal and spatial) approach is a method to avoid interference and still exploit diversity, its complexity is often prohibitive, especially in high mobility environments where fast update rates are necessary. A MIMO RAKE receiver offers the ability to exploit the advantages of the multipath propagation at a reasonable implementation cost. However, this is done at the cost of additional interference.

A basic $M \times N$ MIMO CDMA signal model can be described as follows. The index $k$ denotes one of the $K$ active combined channelization and scrambling codes $a^{(k)}[t]$ and the data symbol $d_{m}^{(k)}$ (unit variance) at the $m$ th transmit antenna. If the symbol power of a single user is normalized, the components of the transmitted signal vector $\mathbf{x}[t]=\left[x_{1}[t], \ldots, x_{M}[t]\right]^{T}$ at discrete time steps $t$, during one symbol period, can be written as

$$
x_{m}[t]=\sum_{k=0}^{K-1} \sqrt{\frac{1}{M}} a^{(k)}[t] / d_{m}^{(k)}
$$

The thermal noise and the intercell interference [treated as additive white Gaussian noise (AWGN)] are summarized in the noise vector $\eta[t]=\left[\eta_{1}[t], \ldots, \eta_{N}[t]\right]^{T}$. The signal received at the receive antenna $n$ is

$$
y_{n}[t]=\sum_{m=0}^{M-1} \sum_{k=0}^{K-1} \sum_{l=0}^{L-1} \sqrt{\frac{1}{M}} a^{(k)}\left[t-\tau_{l}\right] d_{m}^{(k)} \alpha_{n, m}^{(l)}+\eta_{n}[t]
$$

and $\alpha_{n, m}^{(l)}$ is the complex channel response of the $l$ th path between transmit antenna $m$ and receive antenna $n$, where $L$ is the number of multipath components. 
A straight forward RAKE receiver with $L_{r}$ fingers is used to resolve the multipath components (i.e., $L_{r}$ is assumed to be the number of resolvable paths). Without a loss of generality the despreading of a single user $k=0$ with code $a^{(0)}[t]$ is analyzed. Integrating over a symbol period and sampling the outcome of the correlators leads to the following expression for a set of softsymbol components at the received antenna $n(n=1, \ldots, N)$, and the RAKE finger $f\left(f=1, \ldots, L_{r}\right)$, corresponding to the (discrete) delays $\tau_{f}$ on which the actual MIMO detection will be performed

$$
\begin{aligned}
r_{n}, f & =\sum_{t=0}^{S_{F-1}} y_{n}\left[t+\tau_{f}\right]\left(a^{(0)}[t]\right)^{*} \\
& =r_{n, f}^{S}+r_{n, f}^{S I}+r_{n, f}^{\mathrm{MAI}}+\sum_{t=0}^{S_{F-1}} \eta_{n}[t]\left(a^{(0)}[t]\right)^{*}
\end{aligned}
$$

where $S_{F}$ is the spreading factor of the desired user. While multipath helps to combat fading and adds diversity for the MIMO detection, it also adds a significant amount of self interference $r_{n, f}^{S I}$ and intracell MAI $r_{n, f}^{\mathrm{MAI}}$. With term $A_{0, k}[\tau]$ denoting the cross-correlation function between the code $a^{(0)}[t]$ and $a^{(k)}[t]$ and $A_{0,0}[\tau]$ the auto-correlation function of $a_{0}[t]$, the terms in (4) are defined as

$$
\begin{aligned}
r_{n, f}^{S} & =\sum_{m=0}^{M-1} \sqrt{\frac{1}{M}} \underbrace{A_{0,0}[0]}_{S_{F}} \alpha_{n, m}^{(f)} d_{m}^{(0)} \\
r_{n, f}^{S I} & =\sum_{m=0}^{M-1} \sum_{l \neq f}^{L-1} \sqrt{\frac{1}{M}} A_{0,0}\left[\tau_{f}-\tau_{l}\right] \alpha_{n, m}^{(l)} d_{m}^{(0)} \\
r_{n, f}^{\mathrm{MAI}} & =\sum_{m=0}^{M-1} \sum_{k=1}^{K-1} \sum_{l \neq f}^{L-1} \sqrt{\frac{1}{M}} A_{0, k}\left[\tau_{f}-\tau_{l}\right] \alpha_{n, m}^{(l)} d_{m}^{(k)} .
\end{aligned}
$$

The channelization codes are orthogonal and, therefore, $A_{0, k}[0]=0, k \neq 0$. Note that as opposed to a narrowband system, the number of received signals is not only determined through the number of antennas, rather, $N \times L_{r}$ signal terms are available to the MIMO detector. They can be interpreted as virtual antennas [22]. In the following, the time index $t$ is omitted. The output of the MIMO RAKE receiver is

$$
\mathbf{r}=\mathbf{H d}+\mathbf{n}
$$

where $\mathbf{r}=\left[r_{1,1} \cdots r_{1, L_{r}} \cdots r_{N, 1} \cdots r_{N, L_{r}}\right]^{T}$ and $\mathbf{d}=$ $\left[d_{1}^{(0)} \cdots d_{M}^{(0)}\right]^{T}$ is the transmitted data vector. The $\left((n-1) L_{r}+l\right)$ th row and $m$ th column element of the MIMO channel matrix element is $h_{\left((n-1) L_{r}+l\right), m}=$ $\sqrt{1 / M} A_{0,0}[0] \alpha_{n, m}^{(l)}$. The matrix $\mathbf{H}$ is $N L_{r} \times M$ dimensional. The vector $\mathbf{n}$ models the noise corresponding to the self interference, MAI and background AWGN (we assume that $\left.E\left[\mathbf{n n}^{H}\right]=N_{0} \mathbf{I}\right)$.

Further elaboration of the above equations shows that the signal-to-interference ratio (SIR) is directly proportional to $S_{F}$, and it is inversely proportional to the number of the users (i.e., codes) in the cell (cell loading) [22]. This insight strongly suggests that the spatial multiplexing should be the preferred means to increase data rates as opposed to the usage of lower spreading factors, multilevel or multicode transmission (as in the case of

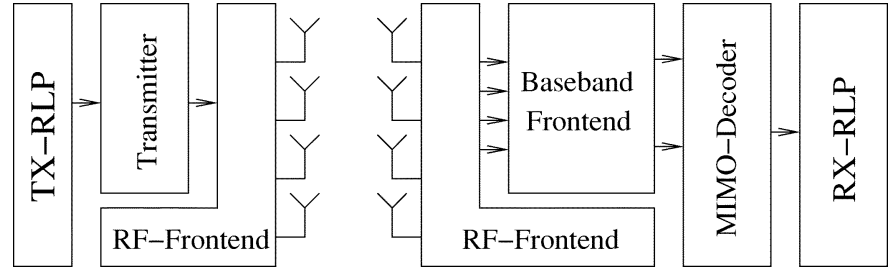

Fig. 2. MIMO system overview.

HSDPA [23]). A similar conclusion in a slightly different context was drawn from simulations in [24], where it was stated that the use of multiple antennas should be preferred over higher order modulation schemes to increase throughput.

\section{RECEIVER DESIGN}

The receiver in this project is a strict feed-forward design. Selecting such a structure comes with many advantages. In particular, feedback loops often used for control signals disappear and, thus, potential instabilities. A further advantage is that at the time of the initial system investigation the final hardware platform does not have to be well defined. A feed-forward structure is easy to partition since cutting out functional blocks does not result in cutting feedback control signals. Such signals would have to undergo an additional delay corrupting the stability of the system due to the interfaces resulting from partitioning. As illustrated in Fig. 2, it consists of a radio frequency (RF) front end, an extended code-division multiple-access (CDMA) baseband processing unit (based on a RAKE receiver architecture), and a MIMO detector stage. The RF and analog front end deliver four time oversampled baseband signals from each of the receive antennas. The baseband processing element performs despreading. A resolution of up to four multipath components, as described in the previous section (four RAKE fingers per receive antenna), has been realized. In this $4 \times 4$ system, each symbol consists, therefore, of 16 soft-symbol components (i.e., 16 virtual antennas). The baseband processor also performs the pilot assisted channel estimation, using the four $\mathrm{CPICH}$ channels of the system [2]. The channel state information at the chosen finger positions is also forwarded to the MIMO detector.

\section{A. MIMO UMTS Baseband Front End}

The baseband front end is an extension of a UMTS downlink receiver front end [16], [17]. It receives samples from the analog-to-digital converters in the RF front end, extracts timing information, and converts the samples into soft symbols and channel information presented to the MIMO detector. Its major blocks and their connections are shown in the block diagram in Fig. 3 and are described in more detail below.

1) Preprocessing: The preprocessing stage receives four complex data streams, one from each of the four receive antennas. Each data stream is four times over-sampled and is initially 14 bits wide. The first processing step reduces the dynamic range such that words are represented by eight bits, thereby lowering the complexity of the remaining parts of the receiver. This reduction is achieved through a digital automatic gain control (AGC). Thereto the average received power is 


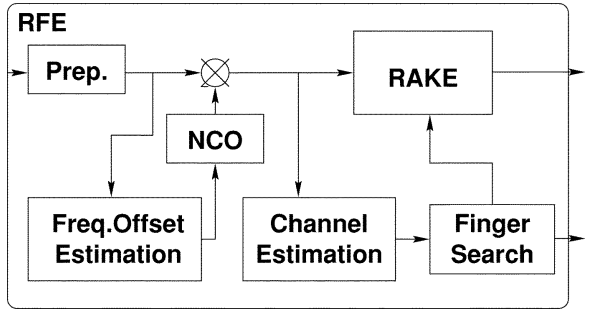

Fig. 3. Baseband front end.

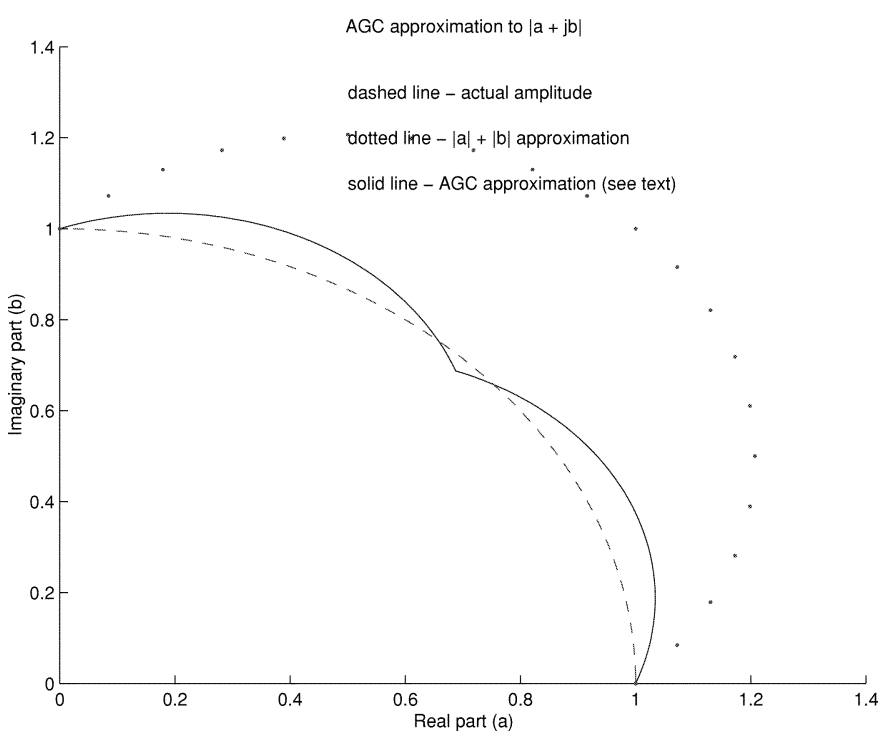

Fig. 4. AGC approximation to $|\mathrm{a}+\mathrm{jb}|$.

estimated separately for each receive antenna. This computation is done in two steps: first a block average over 1024 samples is computed. To avoid costly complex multiplications the magnitude $\left\|y_{n}\right\|$ is approximated as

$$
\frac{3}{8}\left(\left|\Re\left\{y_{n}\right\}\right|+\left|\Im\left\{y_{n}\right\}\right|\right)+\frac{5}{8} \max \left(\left|\Re\left\{y_{n}\right\}\right|,\left|\Im\left\{y_{n}\right\}\right|\right) .
$$

Such approximation is very well-suited for low complexity realization. Its effectiveness is shown in Fig. 4. Herein, the above equation is plotted for $e^{j \theta}$ over a range of angles (solid curve). For comparison, the common approximation $\left|\Re\left(e^{j \theta}\right)\right|+\left|\Im\left(e^{j \theta}\right)\right|$ is also shown (dotted curve).

In the current implementation, the AGC value is independently computed for each of the four receive antenna complex sample streams. As will be seen later, the channel estimation is performed over an integer number of pilot periods. Provisions were made in the design of the multichannel AGC to limit gain adjustments to certain predefined time instances, e.g., channel estimation periods. This technique can ensure that AGC action will not corrupt the MIMO detection algorithms.

Furthermore, concerns were raised during the testing of this system as to whether it was better to allow the AGC to apply independent gain values to each data stream, or to force the multichannel AGC to apply a common gain coefficient to all data streams. While individual gain values provide the best mapping of the 14 bit samples into the internal 8 bit word size, severe noise injection can occur when a single antenna experiences a deep radio fade. The current implementation allows for independent gain values on each data stream. When activated the
AGC was experimentally verified to level the input signals over a 30-dB dynamic range. It is noted, however, that the performance data reported in this article were taken with the AGC gains fixed, effectively implementing the common gain value method.

The square-root-raised-cosine (SRRC) matched filtering with roll-off $\alpha=0.22$ is performed after the digital AGC. This is important for complexity reasons and to remove out-of-band quantization noise from the AGC in the four-time oversampled signal.

2) Frequency Offset Estimation: In the next processing step, large systematic frequency offsets which are caused by RF-oscillator mismatches between the transmitter and receiver are removed. This is necessary as carrier frequencies of about $2 \mathrm{GHz}$ typically have offsets that range up to a few kilohertz and are, therefore, much too high to be tracked by the channel estimator. The offset is estimated through the phase variation of a single received pilot signal after despreading. This scheme can be implemented very efficiently as described in [18]. As a fairly static offset is assumed, an extensive averaging can be used to obtain a reliable estimate which was implemented. Further improvement in the estimate is obtained by taking advantage of spatial diversity at the receiver (e.g., combining of energy from the four receiver antennas). The estimated frequency offset is used to compensate the received signal via a numerically controlled oscillator and a complex rotator. A feed forward scheme is employed: that is to say that the frequency estimator continuously observes the input signal while the derotated signal is fed to the channel estimator and RAKE receiver.

3) MIMO Channel Estimation: The channel estimator is the most complex part of the front end in terms of the number of operations. As opposed to a $1 \times 1$ link, 16 channels need to be estimated in a $4 \times 4$ system, with four RAKE fingers per receive antenna. In our implementation, a maximum usable channel length of 16 chips (or $4.17 \mu \mathrm{s}$ ) was assumed. The estimation is done by chip-matched filtering of each of the four received sequences independently with all training sequences. If the pilot codes of the transmit antennas are restricted to the same branch in the OVSF tree, this estimation procedure can be implemented very efficiently using a combined FIR and correlator approach. The optimum averaging length for the channel estimation varies, depending on the Doppler frequency and on the received SNR. In a high SNR environment, shorter averaging allows for a higher Doppler shift, while in a low SNR environment more averaging is required to obtain good channel estimates. The implemented solution allows performing the averaging over a period of one, two, four, or eight pilot symbols. In the following, $\hat{\mathbf{H}}$ denotes the estimate of the channel $\mathbf{H}$ used in (8).

The performance of one of the 16 channel estimators using an averaging over four pilot symbols is shown in Fig. 5. A three-ray multipath signal was applied between the transmitter and the receiver using a channel emulator. The three paths are clearly visible in this figure with the expected relative time delays and (voltage) amplitudes. Also note that this measurement was taken with the receiver root raised cosine filter disabled, which increases the observed sinc response on either side of the finger correlations. Since the channel estimators coherently sum over 


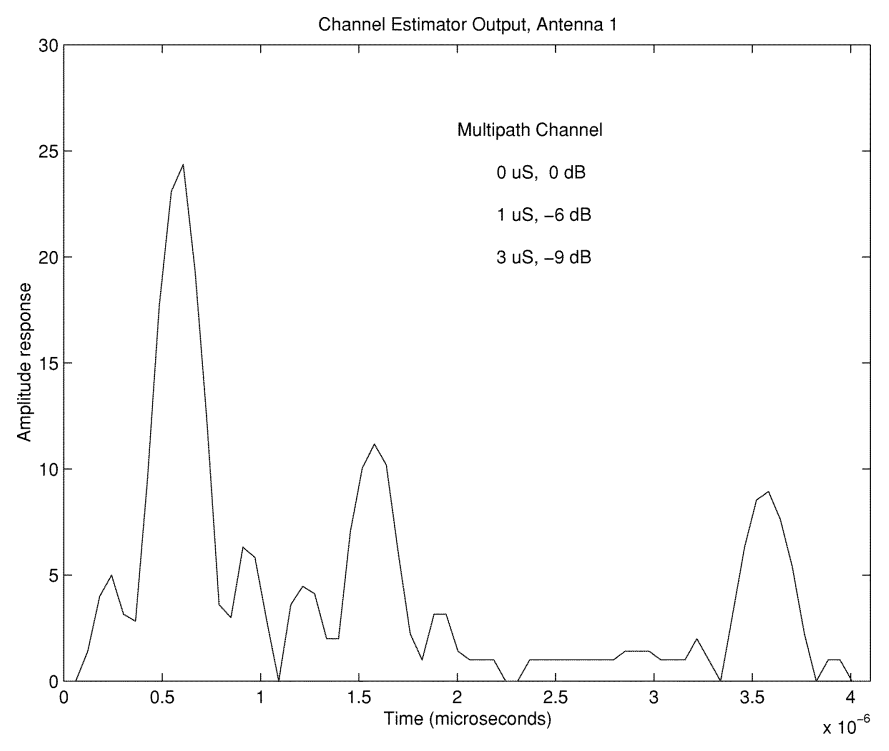

Fig. 5. Channel estimator output.

a period of 1024 chips, the coding gain of this filter is expected to be $30 \mathrm{~dB}$. This is roughly observed by comparing the magnitude of the strongest finger correlation $(25$ units at $0.6 \mu \mathrm{s})$ with the background level (1 unit at $2.6 \mu s$ ).

4) Finger Searcher: The channel estimates are passed on to the finger searcher. This block performs two operations: 1) find the best finger positions for the RAKE receiver and 2) extract the corresponding channel coefficients. The finger positions are assumed to occur with identical path delay for all the 16 subchannels. As the antenna spacing is very small compared to the resolution of the sampled signal this is a reasonable assumption. An approximation of the common power profile to determine the delays of the dominant taps in the channel was used by a simple noncoherent combining of all subchannels

$$
\sqrt{P_{\hat{H}}} \approx \sum_{n=0}^{N-1} \sum_{m=0}^{M-1}\left|\Re\left\{\hat{h}_{n, m}\right\}\right|+\left|\Im\left\{\hat{h}_{n, m}\right\}\right| .
$$

The delays of the four largest values are selected as finger positions. However, a minimum distance of $3 / 4$ chips between any two fingers is always preserved, to avoid correlation from the SRRC filter. The finger positions are passed on to the RAKE receiver. In the next processing step, the corresponding channel coefficients need to be extracted from the channel estimates (i.e., the MIMO channel estimate $\hat{\mathbf{H}}$ is to be created). To achieve this, two approaches differing in their timing relation to the extraction of the finger position were investigated (Fig. 6).

The first approach extracts the coefficients of the $\hat{\mathbf{H}}$-matrix from the same channel estimate which is used to find the positions of the fingers. The advantage of such a scheme is the immediate availability of the channel state information and, thus, the MIMO detector can start with the decoding immediately when a soft symbol is received. The second (delayed) method delays the extraction of the channel coefficients for a set of finger positions until the next channel estimate is available. The estimate is, therefore, obtained concurrently with the despreading of the corresponding soft symbols. This leads to an improved correlation between the estimated and the observed coefficients during the transmission and is especially

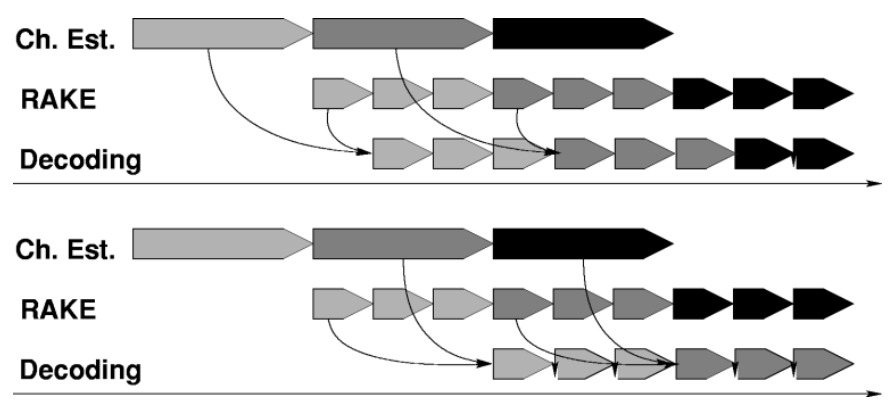

Fig. 6. Instantaneous and delayed channel estimation timing.

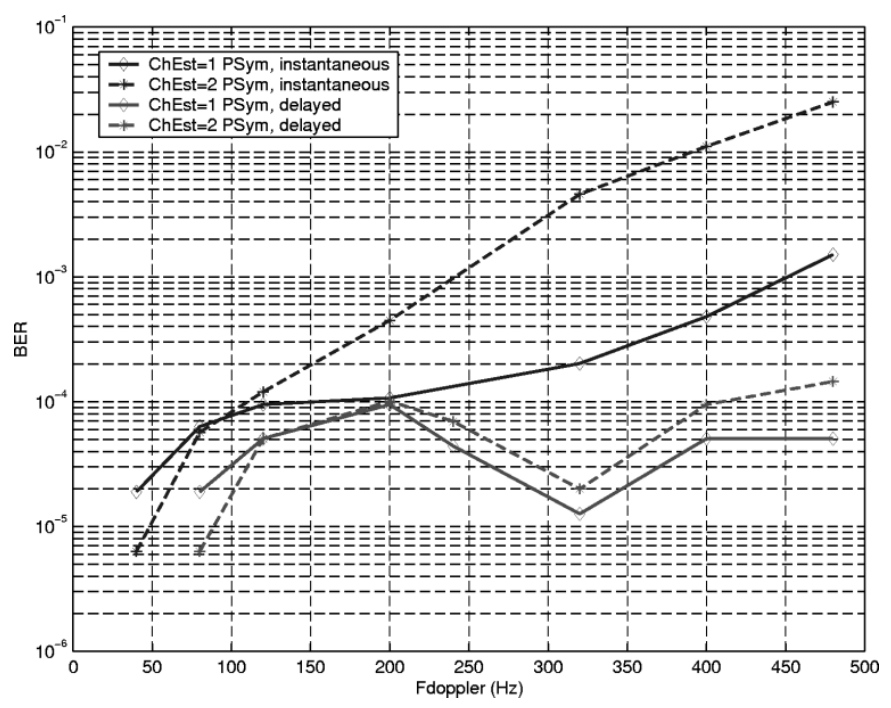

Fig. 7. Influence of the channel estimation method on the BER.

advantageous in high-mobility scenarios. The disadvantage of this approach is that it requires large FIFOs between the front end and the MIMO detector. The performance of the two approaches is compared in Fig. 7. It shows simulation results for a $4 \times 4$ system using the maximum-likelihood (ML) detector with a flat Rayleigh-fading channel. The results are presented for estimation performed over one or two pilot periods. No noise is added and under ideal conditions with perfect channel knowledge no bit errors would be expected. The given curves, therefore, reflect the resulting error floor caused by the noise inferred through imprecise channel estimates. It can be seen that for the undelayed channel estimation the performance degrades even at the Doppler shifts of $50 \mathrm{~Hz}\left(27 \mathrm{~km} / \mathrm{h} @ f_{c}=2 \mathrm{GHz}\right)$. Beyond such Doppler the performance degradation depends strongly on the averaging length (averaging over more pilots might be required in low SNR environments). With the delayed estimation procedure performance only starts to degrade at about $100 \mathrm{~Hz}$ and remains mostly constant up to Doppler shifts of about $500 \mathrm{~Hz}$. In the following, we apply the delayed estimation procedure.

In Fig. 8, the simultaneous operation of all four finger tracker outputs is observed. In this case, four multipaths were applied to the receiver with the following characteristics:

- ray 1: $0.00 \mu s$ delay, 0 -dB power (tap index 7 , reference time and power for other rays);

- ray 2: $0.75 \mu s$ delay, $-3-\mathrm{dB}$ power (tap index 19, Raleigh fading, $20 \mathrm{~Hz}$ Doppler); 

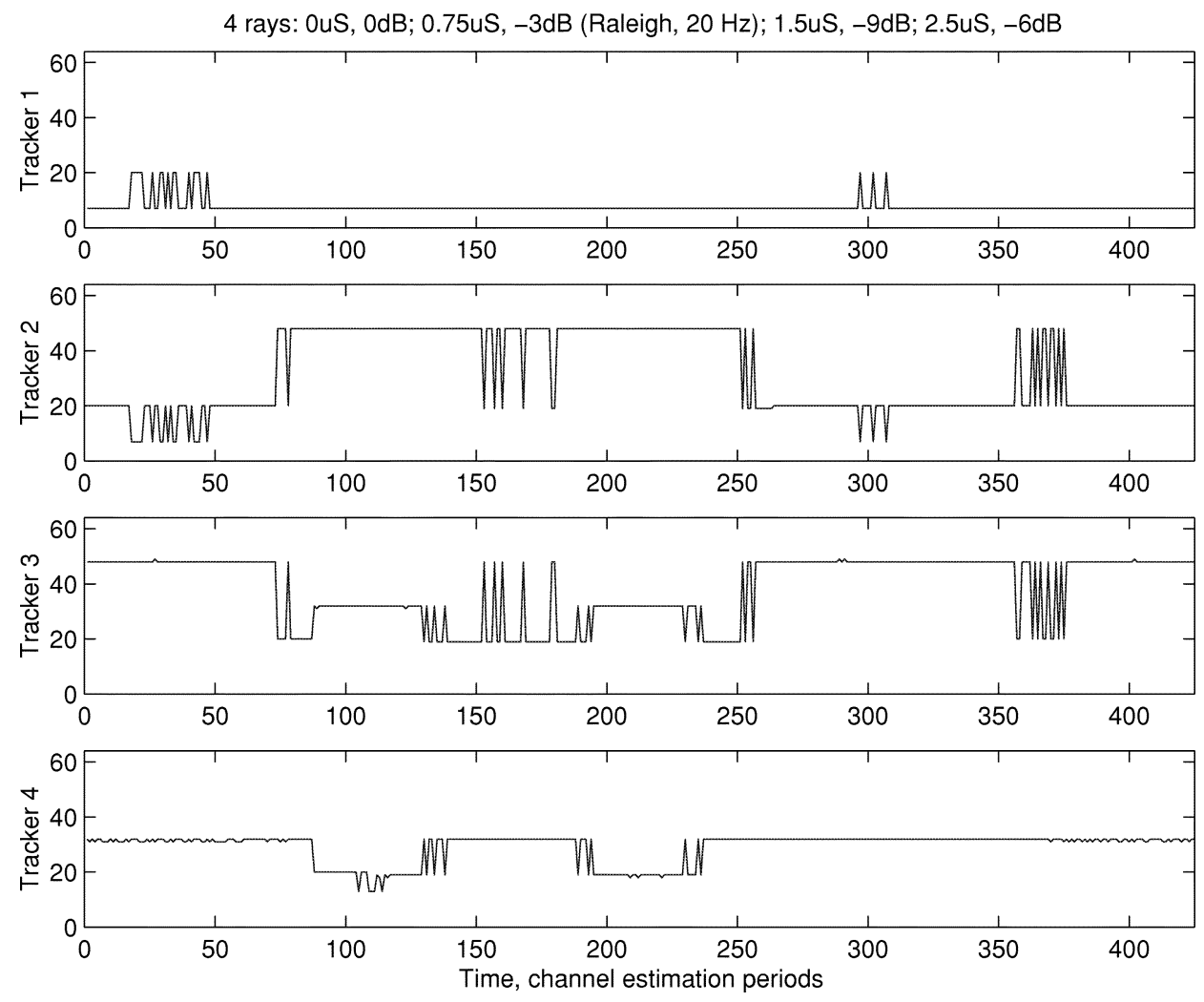

Fig. 8. Finger searcher output for multiple ray scenario.

- ray 3: $1.50 \mu s$ delay, $-49-\mathrm{dB}$ power (tap index 32);

- ray 4: $2.50 \mu s$ delay, -6-dB power (tap index 48).

The outputs are ordered such that tracker 1 provides the index for the strongest multipath, tracker 2 provides the index for the next strongest multipath, tracker 3 provides the index for the third-strongest multipath, while tracker 4 provides the index for the fourth strongest multipath.

The recorded data begins just prior to an up fade of ray 2, with each tracker locked onto the nominal multipath indices (e.g., 7, 19, 48, 32). Around channel estimation period 20 an up fade begins such that ray 2 is stronger than ray 1 ; consequently, the outputs of tracker 1 and tracker 2 switch places. This up fade is not strong enough to change the power ordering of ray 3 and ray 4 relative to the first two rays. However, around channel estimation period 75 a fade begins on ray 2 . The power on ray 2 is first weaker than ray 3 and soon afterwards ray 4 (time 90) also becomes weaker. As such tracker 2 reports the index of ray 4 , tracker 3 reports the index of ray 3 , and tracker 4 reports the index of fading ray 2. Around time 110 the fade becomes so deep that tracker 4 briefly reports the index for a correlation side lobe of ray 1 (the strongest ray). Note that for these tests the SRRC was disabled, so the correlation side lobes were stronger than experienced in normal operation.

5) RAKE Receiver: The MIMO RAKE provides a maximum of four fingers per receive antenna. Temporal positions of the fingers are determined in the finger searcher procedure that is previously described. The RAKE receiver performs the despreading and generates soft symbols [16 complex soft symbols are sent to the MIMO detector, corresponding to the vector $\mathbf{r}$ in (8)]. It is implemented using a set of synchronized correlators in combination with a tapped delay line. As opposed to a realization with independent, delayed correlators this greatly facilitates the finger management and avoids the loss of samples or entire symbols when fingers move [18].

6) Performance Evaluations of the Baseband Front End: In addition to previously described results, in order to assess the performance of the system, a series of measurements were conducted. Most of the measurement techniques have been equivalent to the techniques applied in conventional single-input-single-output (SISO) wireless systems. For example, error vector magnitude (EVM) measurements were conducted. The EVM performance is measured for different number and arrangements of the transmit and receive antennas using cabled and over-the-air measurements. The EVM measurements correspond to the despreaded signal $\left(S_{F}=32\right)$ and the above channel estimation using 1024 chip long pilot period. The EVM is used to assess the effective SNR in the system prior to the MIMO detection. It is effected by the quality of the transmitter, receiver RF front end, digital baseband preprocessing, MIMO channel estimation, and the overall noise in the system. Therefore, it represents a comprehensive measure of the system performance. In the case of the cabled RF connection between the transmitter and the receiver, the EVM that corresponds to an SNR of $25 \mathrm{~dB}$ or higher has been consistently observed. The SNR is estimated based on the dispersion of the received constellation points.

Furthermore, it was decided that a testing technique based on the emulation of the keyhole effect [26] should become an integral part of the MIMO system performance evaluations. Thereto all four RF transmitter outputs are combined together (using 4:1 


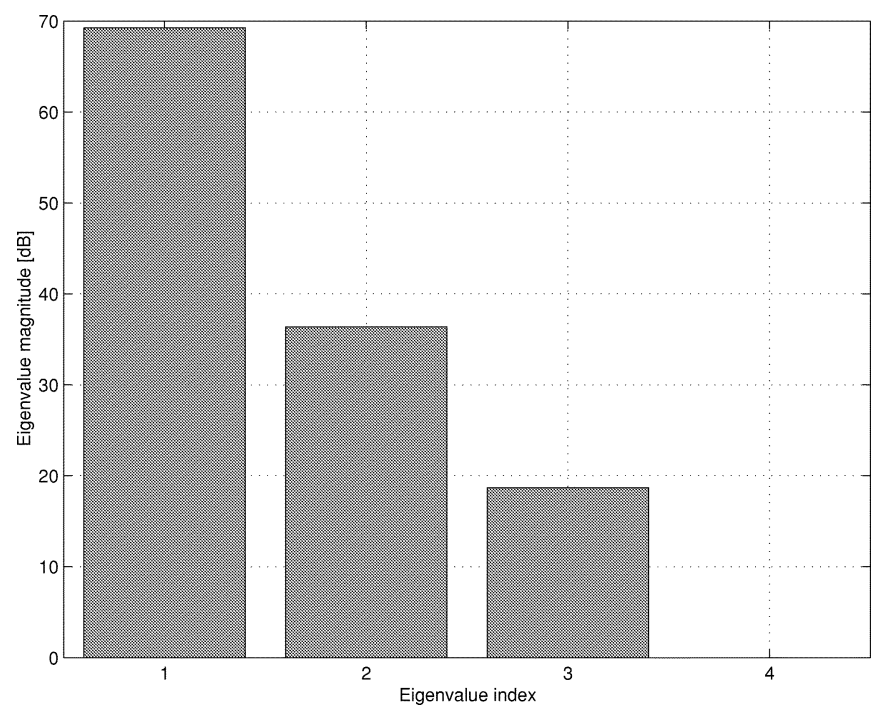

Fig. 9. Eigenvalue profile of the keyhole test, normalized to the weakest eigenvalue.

combiner), and using a single cable, the signal is brought to the receiver. The signal is then fed to each of the receiver RF inputs (using a 1:4 splitter). Having constructed the above system, the initial four dimensional system has collapsed to a single dimensional system (by combining all RF outputs into a single signal) which is denoted as the keyhole effect. Estimating the channel response of the keyhole, using the previously described estimation procedure (1024 chip long pilot period), the estimate of the channel matrix $\mathbf{H}$ has been obtained. In the case of the ideal system, only one nonzero eigenvalue of the $\mathbf{H H}^{H}$ should exist (the channel has single dimension). Because of the noise in the transmitter and receiver and imperfection during the channel estimation, more than one eigenvalue is nonzero. In this particular case, for a system with the acceptable performance, the strongest eigenvalue should be significantly stronger than the other eigenvalues. As seen in Fig. 9, our system provides approximately $32 \mathrm{~dB}$ difference between the strongest and second strongest eigenvalue (single RAKE finger per receive antenna is used). The eigenvalues are normalized with respect to the weakest eigenvalue (the forth one). This result shows that the system is built well, having a low additive noise.

\section{B. MIMO Detection}

In such a feed-forward structure with no explicit interference cancellation the MIMO detector stage is practically identical to the MIMO detection in a narrowband system. The corresponding signal model is given in (8). The well-known algorithms are described in the following.

- ZF receiver:

$$
\hat{\mathbf{d}}=\operatorname{Quantize}\left[\left(\hat{\mathbf{H}}^{H} \hat{\mathbf{H}}\right)^{-1} \hat{\mathbf{H}}^{H} \mathbf{r}\right]
$$

where the matrix $\hat{\mathbf{H}}$ is an estimate of the MIMO channel $\mathbf{H}$. It is obtained using the pilot assisted estimation which is previously described. The vector $\hat{\mathbf{d}}$ is the estimate of the transmitted data in (8).

- ZF-VBLAST [22], [27]-[28]: is an iterative decoding method based on what is known from the literature as a down-dating method (see, for example, [29, Ch. 2.7]). Its implementation can be described as follows.

1) Reset the counter $p=1$.

2) Determine the zero-forcing (ZF) detector from the following iterative orthogonalization scheme

$$
\mathrm{m}_{p}^{(i)}=\mathrm{m}_{p}^{(i-1)}-\frac{\left(\left(\mathbf{m}_{p}^{(-1)}\right)^{H} \hat{\mathbf{h}}_{p+i}\right) \hat{\mathbf{h}}_{p+i}}{\left(\hat{\mathbf{h}}_{p+i}^{H} \hat{\mathbf{h}}_{p+i}\right)}
$$

where $\hat{\mathbf{h}}_{j}(j=1, \cdots M)$ is the $j$ th column vector of the matrix $\hat{\mathbf{H}}$. The iteration is executed in $M-p$ steps, i.e., $i=1, \cdots, M-p$, where $\mathbf{m}_{p}^{(0)}=\hat{\mathbf{h}}_{p}$. The ZF detector is $\mathbf{m}_{p}=\mathbf{m}_{p}^{(M-p)}$, corresponding to the last iteration step.

Note that normalization of the ZF detector is not needed because the QPSK modulation is assumed. The ZF detector is the orthogonal component of $\hat{\mathbf{h}}_{p}$ to the space spanned by the interferers $\hat{\mathbf{h}}_{i}, i=p+$ $1, \cdots M$.

3) Project the received vector on the $\mathrm{ZF}$ detector as

$$
\hat{s}_{p}=\mathbf{m}_{p}^{H} \mathbf{r} .
$$

Using the above result decide on the received data. In the case of QPSK modulation $\hat{d}_{p}=\sqrt{1 / 2}\left(\operatorname{sgn}\left(\operatorname{Re}\left(\hat{s}_{p}\right)\right)+j \operatorname{sgn}\left(\operatorname{Im}\left(\hat{s}_{p}\right)\right)\right)$.

4) Cancel sublayer $p$ contribution as

$$
\mathbf{r}=\mathbf{r}-\hat{d}_{p} \hat{\mathbf{h}}_{p} .
$$

5) Increment $p, p=p+1$, and repeat steps 2 to 5 if $p \leq$ $M$, i.e., the above steps are performed repeatedly for all sublayers.

In addition to the above described steps a power ordering of the sublayers is needed, however for the sake of simplicity it is assumed in the description that $\left\|\hat{\mathbf{h}}_{1}\right\|^{2} \geq$ $\cdots \geq\left\|\hat{\mathbf{h}}_{M}\right\|^{2}$.

- MMSE receiver:

$$
\hat{\mathbf{d}}=\text { Quantize }\left[\left(\hat{\mathbf{H}}^{H} \hat{\mathbf{H}}+N_{0} \mathbf{I}\right)^{-1} \hat{\mathbf{H}}^{H} \mathbf{r}\right] .
$$

- MMSE-VBLAST receiver: successively using MMSE with matrix $\hat{\mathbf{H}}$ of decreasing dimension, based on the smallest row norm of $\left(\hat{\mathbf{H}}^{H} \hat{\mathbf{H}}+N_{0} \mathbf{I}\right)^{-1} \hat{\mathbf{H}}^{H}$. Note that this scheme is equivalent to the ZF-VBLAST algorithm for $N_{0}=0$.

- ML receiver: A full search over all possible constellations $\mathbf{d}_{j}$ is performed [24], [30].

$$
\hat{\mathbf{d}}=\arg \min _{\mathbf{d}_{j}}\left(\left\|\mathbf{r}-\hat{\mathbf{H}} \mathbf{d}_{j}\right\|^{2}\right) .
$$

- Iterative cancellation receiver: starting with an initial estimate $\hat{\mathbf{d}}[0]$, the estimate is iteratively improved. A detailed discussion of this procedure can be found in [14].

To assess the performance of these algorithms all of them were implemented in ANSI-C and simulations were carried out. In the first experiment, no multipath components were assumed. The components of the channel matrix $\mathbf{H}$ were all flat Rayleigh fading with a low Doppler frequency. The spreading factor was set to 32 and channel estimation was performed over a period of 


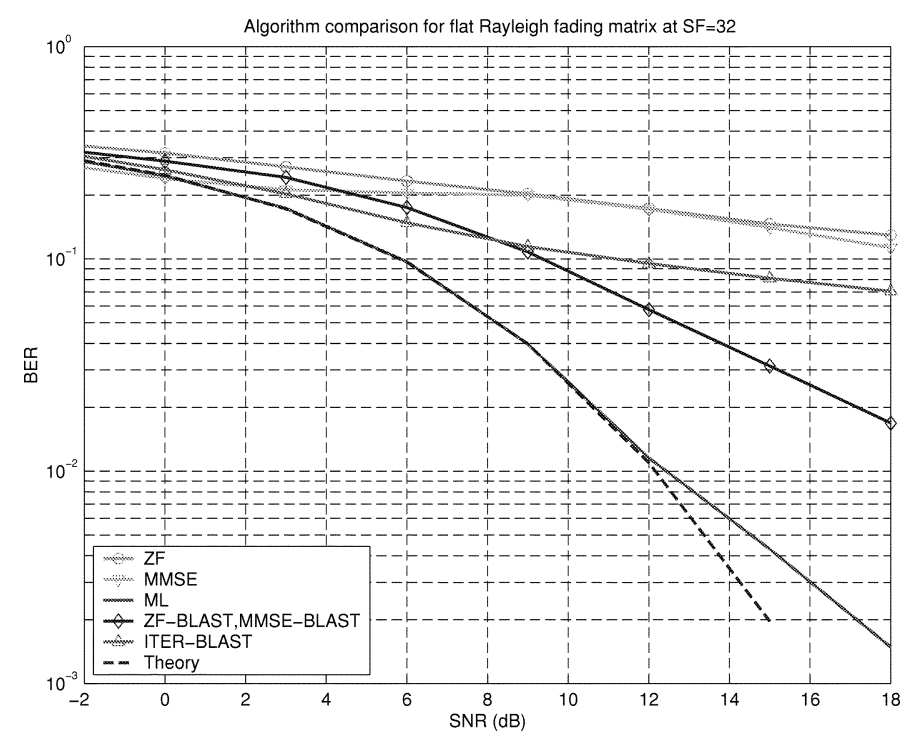

Fig. 10. BER comparison under the flat Rayleigh channel scenario.

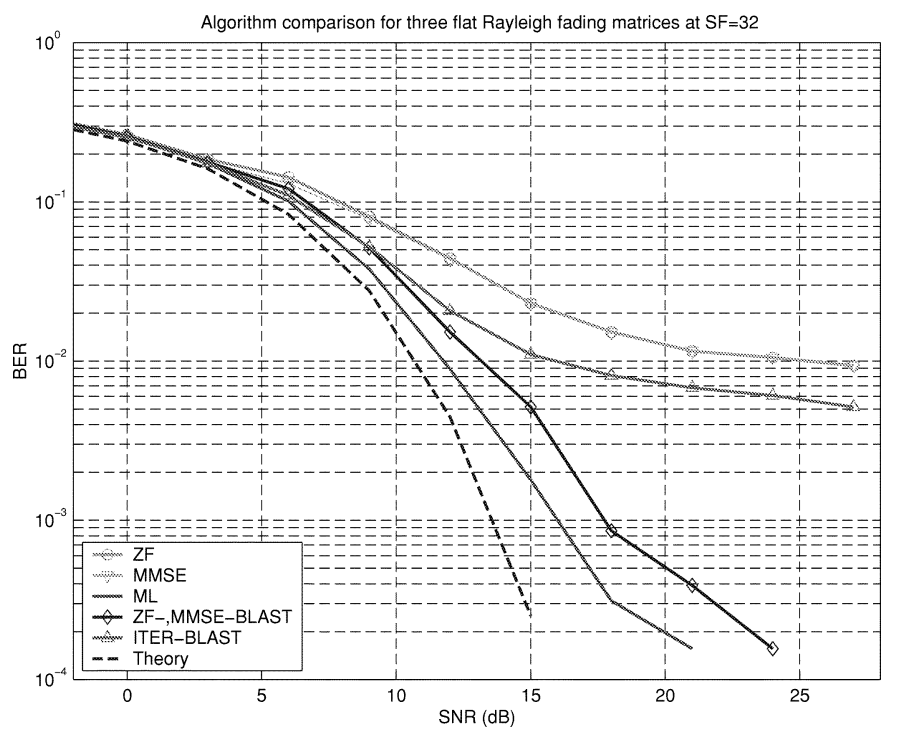

Fig. 11: BER comparison under Rayleigh channel scenario with three paths of equal power.

1024 chips. The results for this scenario are shown in Fig. 10. The ML detector reaches almost the optimum performance predicted, while the ZF-VBLAST algorithm and the pure ZF algorithm exhibit a much lower performance in this case. Regarding the ML scheme this is in full agreement with [24], however, in [24] it is argued that the ZF-VBLAST receiver would result in a poor performance determined by a diversity order of one. As Fig. 10 shows the actual performance is by far not as poor as expected.

The second set of simulations was performed assuming a more realistic propagation model with three equally spaced fading multipath components at delays of $0, T_{C}$, and $2 T_{C}$ (where $T_{C}$ is the chip period). The correlation coefficients, i.e., the variance of the Rayleigh distributions at all path delays were assumed to be identical. Results are given in Fig. 11. In this case, the ZF-VBLAST detector exhibits a comparable performance to the ML detector for lower SNRs while for high SNRs the ML detector delivers a much better BER.
In conclusion of this comparative study, we can state that the ML and ZF-VBLAST schemes outperform the straight forward $\mathrm{ZF}$ detector significantly in most cases. The performance difference between the ML and ZF-VBLAST receiver depends on the nature of the channel and on the SNR. The existence of multipath components in the channel also appears to have a positive effect on the ZF-VBLAST performance. The simulation results will be compared to the measurements further ahead (Section III).

1) Complexity: The difficulty in the implementation of the ML detector is the fact that its complexity grows exponentially with the number of constellations and transmit antennas respectively. However, as opposed to the much less complex ZF-VBLAST algorithm, its implementation is extremely regular and does not require floating-point operations. Together with the advantage offered by the QPSK modulation scheme [31] this allows a number of optimizations based solely on algebraic transforms which lead to a very efficient implementation without sacrificing performance. Table I compares the complexity of the ML and the ZF-VBLAST receiver. The numbers assume a spreading factor of 32 and a channel estimation period of 1024 chips. As multiplications are generally assumed to be more complex than add/compare (ADD/CMP) operations, the overall complexity of the two algorithms turns out to be comparable or even in favor of the ML detector for a reasonably small number of antennas (e.g., $4 \times 4$ ) and smaller constellations. For an ASIC or FPGA implementation this advantage is even more pronounced.

\section{Implementation: Experimental Platform and Rapid Prototyping Design Methodology}

All essential components of the system were initially implemented in $\mathrm{C}$ using the rapid prototyping methodology presented in [16]. An initial, purely behavioral model was gradually refined and optimized. The same $C$ code was used to carry out performance simulations, as well as the functional verification of the system prior to its actual implementation. Subsequently, the code was targeted to a flexible prototyping platform equipped with a XILINX Virtex2-6000 FPGA, a TI-C67 DSP, and a Motorola 8260 PowerPC.

For the transmitter, this platform was integrated as a mezzanine board into a prototype of the Lucent $3 \mathrm{G}$ base station, (OneBTS), which provides the radio interface for four antennas and the clocking. The radio link protocol (RLP) was integrated on the PowerPC running Linux, and the transmitter was mapped to an XILINX FPGA (Virtex2).

The receiver was realized as a stand-alone version of the same prototyping platform. Its physical layer consists of a custom made RF front end. The baseband front end was mapped to the FPGA and the actual MIMO-detector was realized in the DSP. The PowerPC handles the simplified RLP which provides an interface from the physical layer to the TCP/IP stack on a Linux host-platform to enable UDP and TCP connections over the air. Two versions of the receiver were realized: 1) the initial experimental setup consisting of off-the-shelf evaluation boards from SUNDANCE and laboratory equipment (for clock generation) and 2) a more recent stand alone custom-made compact realization as shown in Fig. 16 together with the OneBTS. 
TABLE I

COMPUTATIONAL COMPLEXITY OF ZF-VBLAST AND ML

\begin{tabular}{c|c|c|c|c|c|c|c}
\hline & & \multicolumn{3}{|c|}{ ZF-VBLAST } & \multicolumn{3}{c}{ ML } \\
\hline & Rate & DIV & MUL & ADD/CMP & DIV & MUL & ADD/Cmp \\
\hline \hline Per Channel Estimate & $\frac{3.84 E 6}{1024} \frac{1}{s}$ & 4 & 264 & 640 & - & 96 & 232 \\
\hline Per Symbol & $\frac{3.84 E 6}{32} \frac{1}{s}$ & - & 160 & 104 & - & 64 & 635 \\
\hline \hline Total & $\frac{O p s}{s}$ & $1.5 \mathrm{M}$ & $20 \mathrm{M}$ & $15 \mathrm{M}$ & - & $12 \mathrm{M}$ & $78 \mathrm{M}$ \\
\hline \hline Total DSP cycles (TI-C67) & $\frac{\text { Cycles }}{s}$ & \multicolumn{3}{|c|}{$140 \mathrm{M}$} & \multicolumn{3}{c}{$102 \mathrm{M}$} \\
\hline
\end{tabular}

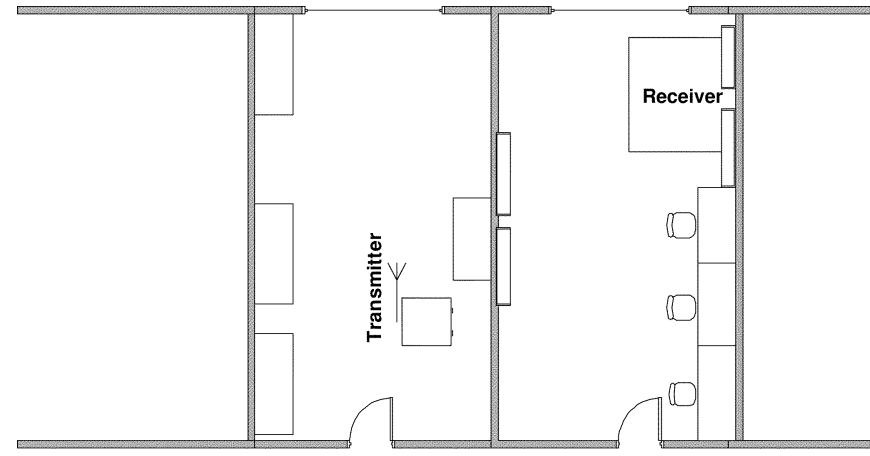

Fig. 12. Measurements environment.

\section{INDOOR OVER-THE-AIR MEASUREMENTS}

In this section, initial indoor over-the-air measurements under mostly static conditions are presented. Capacity curves based on the estimated channel matrix and the received SNR are shown. Further, the initial BER measurement results using the ML and ZF-VBLAST detection are presented. All measurements were taken in an office type environment as depicted in Fig. 12. The transmitter uses $\lambda / 4$ dipole antennas placed along a line with a spacing of about $2 \lambda$ at a height of approximately $3 \mathrm{~m}$. The receiver uses $\lambda$ spaced patch antennas which were placed at various locations in the adjacent room. The receiver antennas were designed to fit within the dimensions of a laptop computer with the four antennas located at the four corners of the back of the LCD display. Each "bowtie" antenna was oriented at $\pm 45^{\circ}$ angles. QPSK modulation was used and no channel coding was applied. A spreading factor of 32 was used with a single active user. Under the assumption of a flat fading environment with no multipath components only one of the four possible fingers at the receiver was turned on. The channel was estimated over a period of 1024 chips or four pilot symbols. In the following, the SNR is defined as the ratio between the total received signal power and the EVM of the multidimensional received signal after despreading. Therefore, the measurement includes the noise inferred in the radios of the transmitter and receiver, the AWGN from the channel and any quantization noise in the receiver.

To make an assessment of the average capacity, the channel $\mathbf{H}$, as well as the SNR were measured at a number of antenna positions. It is assumed that in the static environment channel

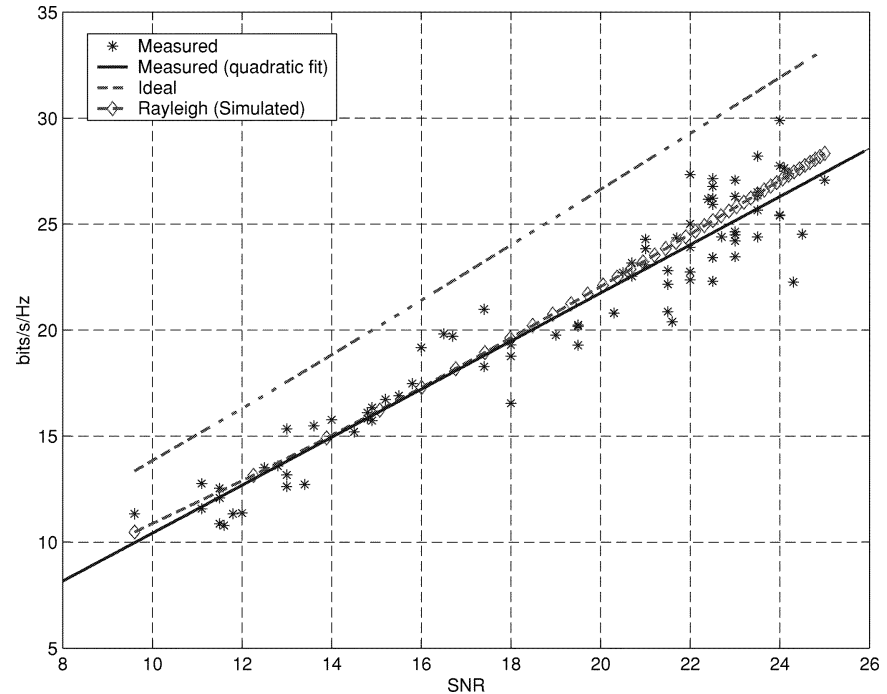

Fig. 13. Channel capacity for $4 \times 4$ configuration.

conditions and SNR remain constant for each particular location throughout the measurement period. The channel matrix at each location needs to be normalized by $\sqrt{\left(\operatorname{trace}\left(\mathbf{H H}^{H}\right)\right) /(M N)}$ before the capacity is extracted following (1). Fig. 13 shows the results and relates them to the capacity when $\mathbf{H}$ is an identity matrix (dashed line) and to the capacity of the idealized (simulated) Rayleigh channel (dotted line). The measured averaged capacity (derived as a quadratic fit) closely follows the idealized Rayleigh channel capacity. This indicates that for the given measurement scenarios, the indoor MIMO channel can be efficiently modeled using the Rayleigh channel model. In the given configuration, the system achieves a throughput of $0.25 \mathrm{~b} / \mathrm{s} / \mathrm{Hz} / \mathrm{channel}$ with a maximum of 31 concurrently active channels. This results in an overall throughput of $7.75 \mathrm{~b} / \mathrm{s} / \mathrm{Hz}$.

Fig. 14 plots the BER versus the received SNR in a spot that was found to provide particularly good performance. The SNR was swept by adding band-limited white noise to the system through a single noise transmitter in close proximity to the receiver. The measured channel estimate was used as input to the static channel model in the MATLAB simulation. The measurements closely follow the simulation results indicating correct functionality of the system and the static nature of the channel. The results are also in good agreement with the previously obtained simulation results for the ML decoder in a flat Rayleigh-fading scenario (Fig. 10). The comparison between the static measurements and the fading simulation 


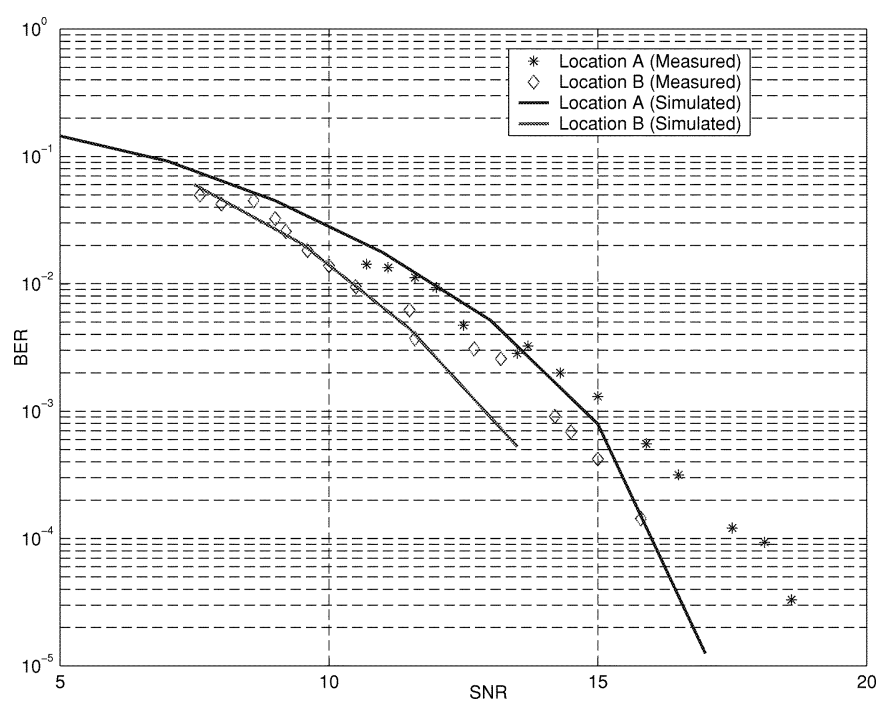

Fig. 14. BER versus SNR.

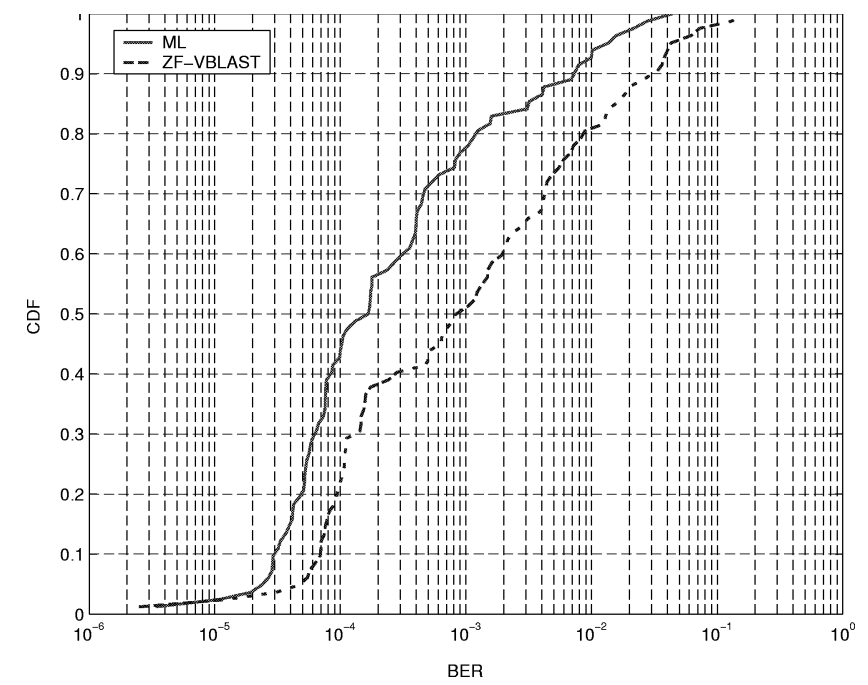

Fig. 15. CDF of the measured BER applying ML and ZF-VBLAST.

model is justified as averaging over multiple measurements and environmental changes lead to a fading behavior of the channel. However the slightly steeper BER curves in the measurements are characteristic of the chosen spot.

The curves in Fig. 15 show the cumulative distribution function of the BER for the ZF-VBLAST and ML detection. As expected from the simulations, the ML detector clearly outperforms the ZF-VBLAST algorithm in this environment.

\section{AN APPLICATION: VIDEO STREAMING}

In order to demonstrate the capabilities and performance of the UMTS MIMO prototype, a video-streaming application was implemented. Publicly available video server and player software from RealNetworks (www.real.com) were used in the demonstration. The end-to-end RTSP (real-time streaming protocol) for data delivery from the RealSystem Server 8 to the RealOne player, was set to use UDP for video transport. A wired ethernet link served as the full-duplex TCP connection between the server and the player for control and negotiation tasks.

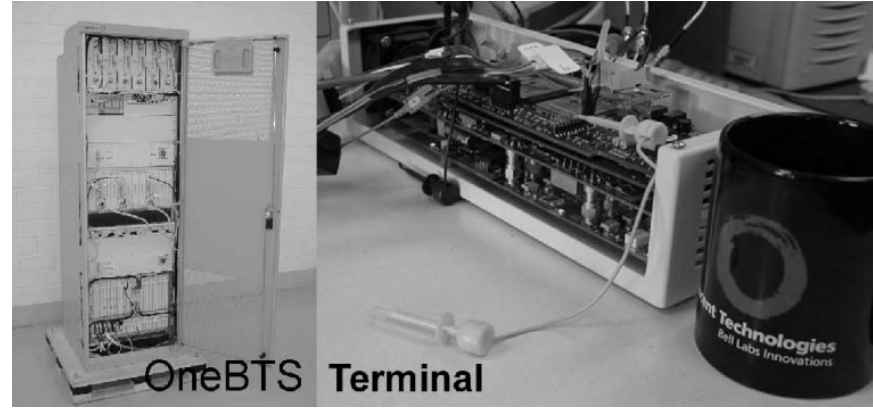

(a)

(b)

Fig. 16. (a) Transmitter in OneBTS. (b) Prototype terminal.

Reliable video transport is accomplished via the use of type-I hybrid ARQ protocol and video packet fragmentation. The two-way communications pipe between TX-RLP and RX-RLP, which are Linux machines external to the radio system, is established through the use of PPP (point-to-point protocol) running over a layer 2 tunnelling protocol (L2TP)-like connection. In the forward direction, from TX-RLP to the RX-RLP, the connection uses the radio link; in the reverse, it uses a wired link. (Video server and video player are running in TX-RLP and RX-RLP machines, respectively). Video packet fragmentation/defragmentation to 512 bytes, in our case, is done in the kernel at the pseudo-TTY interface between PPP and L2TP. L2TP programs at the transmitting and the receiving end provide ARQ functionality, which is based on a sliding window mechanism. Encoding and decoding operations for the $(16,8)$ Hamming channel code are performed in the transmitter and MIMO detector blocks, respectively.

Video content was created using the standard RealProducer package from RealNetworks, at a $450 \mathrm{~kb} / \mathrm{s}$ rate, using SureStream technology but without any optimization for wireless transmission. Over-the-air streaming rates of up to $460 \mathrm{~kb} / \mathrm{s}$ were achieved - considering the $1-\mathrm{Mb} / \mathrm{s}$ raw bit rate and rate-1/2 channel coding, the ARQ layer introduced only a slight reduction in the overall throughput.

\section{CONCLUSION}

In this paper, a prototype implementation of a WCDMA MIMO system is described. Its physical layer is derived from an extension of the UMTS FDD downlink. A theoretical analysis of the system is given to predict its performance under various channel conditions, and the implementation of the critical components of the system is presented. Indoor channel and BER measurements show promising results in good agreement with the expected behavior. Also presented is an outline of a real-time video streaming application using a simple RLP and L2TP-like tunneling, implemented to demonstrate the performance of the system.

\section{ACKNOWLEDGMENT}

The authors gratefully acknowledge the support and encouragement of the many colleagues lead by P. Mankiewich, P. Polakos, G. Rittenhouse, R. Valenzuela, and P. Feder, all from Lucent Technologies. They are particularly grateful to T. Janiszewski and R. Warncke for their readiness to help. 
Their visiting students, M. Guillaud and V. Battier, provided many valuable contributions during their tenure at Lucent Technologies. Thanks also to W. Fichtner and N. Felber from ETHZ for their support for the project and to D. Perels for invaluable discussions and comments.

\section{REFERENCES}

[1] S. Verdú, Multiuser Detection. Cambridge, U.K.: Cambridge Univ. Press, 1998.

[2] H. Holma and A. Toskala, WCDMA for UMTS. New York: Wiley, 2000.

[3] G. J. Foschini, "Layered space-time architecture for wireless communication in a fading environment when using multiple antennas," Bell Labs Tech. J., vol. 1, no. 2, pp. 41-59, 1996.

[4] G. J. Foschini and M. J. Gans, "On limits of wireless communications in a fading environment when using multiple antenna," Wireless Pers. Commun., no. 6, pp. 315-335, 1998.

[5] G. D. Golden, G. J. Foschini, R. A. Valenzuela, and P. W. Wolniansky, "Detection algorithm and initial laboratory results using V-BLAST space-time communication architecture," Electron. Lett., vol. 35, pp. 14-16, Jan. 1999.

[6] P. Kyritsi and D. C. Cox, "Effect of element polarization on the capacity of a MIMO system," in Proc. Wireless Communications and Networking Conf. 2002, vol. 2, Mar. 2002, pp. 892-896.

[7] - "Correlation properties of MIMO radio channels for indoor scenarios," in Proc. 35th Asilomar Conf. Signals, Systems and Computers, vol. 2, Nov. 2001, pp. 994-998.

[8] P. Kyritsi, P. W. Wolniansky, and R. A. Valenzuela, "Indoor BLAST measurements: capacity of multielement antenna systems," Wireless Commun. (Multiaccess, Mobility and Teletraffic), vol. 5, pp. 49-60, Dec. 2000.

[9] P. Kyritsi, D. C. Cox, R. A. Valenzuela, and P. W. Wolniansky, "Effect of antenna polarization on the capacity of a multiple element system in an indoor environment," IEEE J. Select. Areas Commun., vol. 20, pp. 1227-1239, Aug. 2002.

[10] P. Kyritsi, "MIMO capacity in free space and above perfect ground: theory and experimental results," in Proc. Int. Symp. Personal, Indoor and Mobile Radio Communications (PIMRC 2002), vol. 1, pp. 182-186.

[11] D. P. Palomar, J. R. Fonollosa, and M. A. Lagunas, "Capacity results of spatially correlated frequency-selective MIMO channels in UMTS," in Proc. Vehicular Technology Conf. (Fall), vol. 2, 2001, pp. 553-557.

[12] R. Gaspa and J. R. Fonollosa, "Further results on space-time coding for UMTS," in Proc. Vehicular Technology Conf. (Fall), vol. 1, 2001, pp. 183-186.

[13] UMTS-Standard: TS 125.211, TS 125.213 [Online]. Available: http:// www.3gpp.org

[14] M. Rupp, M. Guillaud, and S. Das, "On MIMO decoding algorithms for UMTS," in Proc. 35th Asilomar Conf., Pacific Grove, CA, Nov. 2001, pp. $975-979$.

[15] M. Guillaud, A. Burg, L. Mailaender, B. Haller, M. Rupp, and E. Beck, "From basic concept to real-time implementation: prototyping WCDMA downlink receiver algorithms-A case study," in Proc. 34th Asilomar Conference, Pacific Grove, CA, Oct. 2000, pp. 84-88.

[16] A. Burg, B. Haller, M. Guillaud, M. Rupp, E. Beck, and L. Mailaender, "A rapid prototyping methodology for algorithm development in wireless communications," in Proc. Design, Automation and Test in Europe DATE'01, Munich, Mar. 2001.

[17] M. Guillaud, S. Das, A. Burg, M. Rupp, and E. Beck, "Rapid prototyping design of a $4 \times 4$ blast-over-UMTS receiver," in Proc. 35th Asilomar Conf., Pacific Grove, CA, Nov. 2001, pp. 1256-1260.

[18] A. Burg, E. Beck, M. Rupp, D. Perels, N. Felber, and W. Fichtner, "FPGA implementation of a MIMO receiver front-end for UMTS," in Zürich Seminar, Zürich, Switzerland, Feb. 2002, pp. 8-1-8-2.

[19] J. G. Proakis, Digital Communications. New York: McGraw-Hill, 1995.

[20] M. Médard and R. Gallager, "Bandwidth scaling for fading multipath channels," IEEE Trans. Inform. Theory, vol. 48, pp. 840-852, Apr. 2002.

[21] E. Telatar and D. N. C. Tse, "Capacity and mutual information of wideband multipath fading channels," IEEE Trans. Inform. Theory, vol. 46, pp. 1384-1400, July 2000 .

[22] D. Samardzija, P. W. Wolniansky, and J. Ling, "Performance evaluation of the VBLAST algorithm in W-CDMA systems," in Proc. Vehicular Technology Conf. (Fall), vol. 2, 2001, pp. 723-727.
[23] “Tech. Rep. Third-Generation Partnership Project," Tech. Spec. Group Radio Access Network, 3G TR25.848 V4.0.0 (2001-03).

[24] R. Van Nee, A. Van Zelst, and G. Awater, "Maximum likelihood decoding in a space division multiplexing system," in Proc. Vehicular Technology Conf. (Spring), vol. 1, 2000, pp. 6-10.

[25] W. C. Jakes, Microwave Mobile Communication. Piscataway, NJ: IEEE Press, 1974.

[26] D. Chizhik, G. J. Foschini, M. J. Gans, and R. A. Valenzuela, "Keyholes, correlations, and capacities of multielement transmit and receive antennas," IEEE Trans. Wireless Commun., vol. 1, pp. 361-368, Apr. 2002.

[27] P. W. Wolniansky, G. J. Foschini, G. D. Golden, and R. A. Valenzuela, "V-BLAST: an architecture for achieving very high data rates over richscattering wireless channels," in ISSSE-98, Pisa, Italy.

[28] G. D. Golden, G. J. Foschini, R. A. Valenzuela, and P. W. Wolniansky, "Detection algorithm and initial laboratory results using V-BLAST space-time communication architecture," Electron. Lett., vol. 35, pp. 11-14, Jan. 1999.

[29] T. Kailath, A. H. Sayed, and B. Hassibi, Linear Estimation. Englewood Cliffs, NJ: Prentice-Hall, 1999.

[30] G. Awater, A. Van Zelst, and R. Van Nee, "Reduced complexity space division multiplexing receivers," in Proc. Vehicular Technology Conf. (Spring), vol. 1, 2000, pp. 11-15.

[31] M. Rupp and J. Balakrishnan, "Efficient chip design for pulse shaping," in Proc. Signal Processing Advances in Wireless Communications (SPAWC'99), Annapolis, MD, May 1999, pp. 304-307.

Ali Adjoudani received the M.S. degree in solid state physics and speech and signal processing, and the Ph.D. degree in audio-visual speech recognition both from the Institute National Polytechnique de Grenoble, France.

He joined the research lab of France Telecom (CNET), focusing on highquality speech and music coding. He is a Member of Technical Staff, Bell Labs, Lucent Technologies, Holmdel, NJ. He is currently working with the Wireless Research Lab on signal processing and hardware/software architecture and implementation for CDMA2000 and UMTS

Eric C. Beck received the B.S.E.E. degree from the Pennsylvania State University, University Park and the M.Eng. degree (electrical) from Cornell University, Ithaca, NY.

He is currently Distinguished Member of Technical Staff, Lucent Technologies, Holmdel, NJ, in the Mobility Solutions Business Unit. During his career at Bell Labs, he has also held assignments as a Technical Manager in a variety of technical and product groups. Examples include cellular telephony (handsets and emerging wireless data systems), undersea transmission systems (FEC and EFA monitoring signal processors), network signal processing (FAX compression), and integrated circuit design (X.25 protocol controllers). He currently holds two patents with one pending.

Andreas P. Burg (S'95) was born in Germany in 1975. He received the Diploma degree from the Swiss Federal Institute of Technology (ETH), Zürich, in 2000. $\mathrm{He}$ is currently working toward the Ph.D. degree in electrical engineering in the Integrated Systems Laboratory, ETH.

He worked for six months as an Intern at Bell Labs, Lucent Technologies, Holmdel, NJ. His work is sponsored by Lucent/Bell Labs Research. His interests are multiple antenna systems and VLSI design for wireless communication.

Mr. Burg was awarded the ETH-Medal for his outstanding Diploma-Thesis and the ETH "Willi Studer Award" for graduation as Best in Class.

Goran M. Djuknic received the Diploma and M.S. degrees from the University of Belgrade, Yugoslavia, and the Ph.D. degree from City College, New York, all in electrical engineering.

Since 1995, he has been with Bell Labs, Lucent Technologies, Whippany, NJ, where he worked in the areas of geolocation, satellite communications, wireless multimedia, and innovative approaches to establishing wireless communications services. He currently works on the development and experimental implementation of $3 \mathrm{G}$ wireless systems. 
Thomas G. Gvoth received the B.S. degree in electrical engineering from New Jersey Institute of Technology, Newark, in 1982 and the M.S. degree from Stevens Institute of Technology, Hoboken, NJ, in 1986.

From 1982 to 1991, he worked for the Signal Processing Design Group of the Singer Company, Kearfott Division, Wayne, NJ. In 1991, he joined Cellular Systems of AT\&T, Bell Laboratories, Whippany, NJ. Currently, he is a Member of Technical Staff in the Wireless Advanced Technologies Laboratory, Lucent Technologies, Whippany. His technical interests include DSP and microcontroller hardware/software design for wireless communications.

D. Haessig (M'87-S'93-SM'00) received the B.S. and M.S. degrees in mechanical engineering from Lehigh University, Bethlehem, PA, in 1979 and 1981 , respectively, and the Ph.D. degree in electrical engineering from New Jersey Institute of Technology, Newark, in 1999.

$\mathrm{He}$ is currently a Member of Lucent's Wireless Advanced Technology Lab, Holmdel, NJ, joining in October of 2000. During the 17 years prior, he was employed by BAE Systems, Wayne, NJ, where his work involved development of satellite communication systems, inertial sensors, control design for nigh vision, and simulation analysis of missile guidance systems. From 1981 to 1983 , he was employed by General Dynamics and involved in submarine control.

Salim Manji (S'95) received the B.A.Sc. and M.A.Sc. degrees in electrical engineering from the University of Waterloo, Waterloo, ON, Canada, in 1995 and 1996, respectively. He is currently working toward the Ph.D. degree at WINLAB, Rutgers University, Piscataway, NJ.

During the summers of 1997-1999, he held an internship position with the Wireless Applications Laboratory, Lucent Technologies, Bell Labs, Whippany, NJ. His current research interest is in the area of image transmission over wireless channels.

Michele A. Milbrodt received the B.S. degree in electrical engineering from Lafayette College, Easton, PA, in 1990 and the M.S. degree in materials sciences from Stevens Institute of Technology, Hoboken, NJ, in 1994.

In 1980, she joined AT\&T Bell Laboratories, currently Lucent Technologies, Holmdel, NJ, where she worked on guided-wave optical devices and passive optical components. In 1998, she transferred to the Wireless Research Laboratory, where she is currently working on next-generation wireless systems.

Markus Rupp received the Diploma degree in electrical engineering from FHS Saarbruecken, Germany, and the Universitaet of Saarbruecken, in 1984 and 1988, respectively, and the Doctoral degree (summa cum laude) in acoustical echo compensation from the TH Darmstadt, Germany, in 1993.

He was awarded a DAAD Postdoctoral Fellowship and spent the time, from November 1993 until September 1995 in the Department of Electrical and Computer Engineering, University of California, Santa Barbara, working on a robustness theory for adaptive filters. From October 1995 until August 2001, he has been with Bell Labs, Lucent Technologies (before AT\&T), Wireless Research Lab, Holmdel, NJ, working on wireless phones and implementation issues of wireless modems. In May 1999, he moved to The Netherlands, joining Bell Labs efforts in wireless LANs in Europe. In October 2001, he joined the Faculty of electrical engineering and information technology as a Full Professor at the TU Wien, Vienna, Austria. He is currently involved in rapid prototyping methods for wireless systems, as well as MIMO coding and equalization techniques. $\mathrm{He}$ has more then 90 publications and patents in the fields of adaptive filters and wireless systems.

Dragan Samardzija (M'02) received the B.S. degree in electrical engineering and computer science from the University of Novi Sad, Yugoslavia, in 1996 and the M.S. degree in electrical engineering from Rutgers University, Piscataway, NJ, in 2000. Currently, he is working toward the Ph.D. degree at the Wireless Information Network Laboratory (WINLAB), Rutgers University.

He has been with the Wireless Research Laboratory, Bell Labs, Lucent Technologies, Holmdel, NJ, where he is involved in research in the field of MIMO wireless systems. His research interests include detection, estimation and information theory for MIMO wireless systems, interference cancellation, and multiuser detection for multiple-access systems. Also, he works on implementation aspects of various receiver architectures and implementation platforms.
Arnold B. Siegel (S'80-M'82) received the B.E. degree in electrical engineering from The Cooper Union, New York, in 1981 and the M.S.E. degree in electrical engineering and computer science from Princeton University, Princeton, NJ, in 1983.

For 15 years, he worked at GEC-Marconi Electronic Systems Corporation, Wayne, NJ (now BAE Systems), where he designed software defined radio systems for military applications. In 1998, he joined Lucent Technologies, Holmdel, NJ, where he works on the design of next-generation wireless systems.

Tod Sizer, II (M'93) graduated from Amherst College, Amherst, MA (magna cum laude) and received the M.S. and Ph.D. degrees from the Institute of Optics at the University of Rochester, Rochester, NY.

He is Director of the High-Speed Circuits and Systems Research Department, Bell Laboratories, Lucent Technologies, Holmdel, NJ. His department performs research in high-speed wireless systems, RFIC design, and design of next-generation cellular base stations. During his tenure at Bell Labs, he has performed research in wired and wireless home networking, fixed wireless loop systems, video watermarking technologies, optical computing and switching systems, and high power laser design. He is a Member of Technical Staff in Lucent's role as a promoter in the Bluetooth Special Interest Group (SIG). His responsibilities in the SIG include being Chair of the Coexistence Working Group. He is the author of 34 patents, 16 patents pending, and over 50 refereed publications.

C. Tran received the B.S. and M.S. degrees in electrical engineering from New Jersey Institute of Technology, Newark, NJ.

$\mathrm{He}$ is a Member of High-Speed Circuits and Systems Research Department, Bell Laboratories, Lucent Technologies, Holmdel, NJ. His primary technical areas include antennas and mobile radio.

Susan Walker received the B.S. degree from Rutgers University, Piscataway, NJ, in 1984 and the M.S. degree in material science and engineering from Stevens Institute of Technology, Hoboken, NJ, in 1988.

She joined AT\&T Bell Laboratories, Lucent Technologies, Holmdel, NJ, in 1984, working initially on monolithic integrated optics. In 1994, she joined the Wireless Research Division, which in 1996 spun off with Lucent Technologies. Currently, she is a Member of Technical Staff responsible for implementing next-generation wireless solutions.

Stephen A. Wilkus (M'83) received the B.S. degree in physics and the M.S.E.E. degree from the University of Illinois, Urbana, in 1981.

After working at RF Monolithics, he joined Bell Laboratories (then part of AT\&T), Lucent Technologies, Holmdel, NJ, in 1986 as a Researcher and Developer of surface acoustic wave devices used in undersea cable and wireless LANS. This interest in wireless communication lead to work on wireless standards and regulatory work in the AT\&T Chief Architect's Office. In 1991, he spearheaded the development of a wireless electronic shelf label system currently sold by the millions by NCR (also once a part of AT\&T). He currently Supervises a team concentrating on cost effective implementation of advanced wireless technologies.

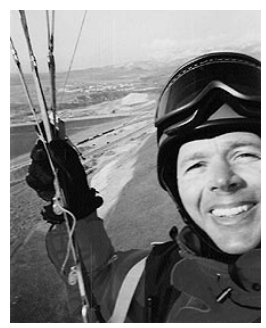

Peter W. Wolniansky received the B.S.E.E. and M.S.E.E. degrees from Boston University, Boston, MA, in 1983 and 1986, respectively.

He performed system tests on the HAWK missile system for Raytheon Corporation, Bedford, MA, from 1983 to 1984 and studied optical data storage for Sony Corporation, Tokyo, Japan, from 1986 to 1987. Since 1988, he has been with Lucent Technologies, Bell Labs Innovations, Holmdel, NJ, as a Member of Technical Staff. His duties have included system engineering of modem networks, millimeter radio design, and propagation studies. His recent activities include multichannel radio design and field studies using antenna arrays. 